\title{
On the chirality of a discrete Dirac-Kähler equation
}

\author{
Volodymyr Sushch \\ Koszalin University of Technology, \\ Sniadeckich 2, 75-453 Koszalin, Poland \\ e-mail: volodymyr.sushch@tu.koszalin.pl
}

October 21, 2018

\begin{abstract}
We discuss a discrete analogue of the Dirac-Kähler equation in which chiral properties of the continual counterpart are captured. We pay special attention to a discrete Hodge star operator. To build one a combinatorial construction of double complex is used. We describe discrete exterior calculus operations on a double comlex and obtain the discrete DiracKähler equation using these tools. Self-dual and anti-self-dual discrete inhomogeneous forms are presented. The chiral invariance of the massless discrete Dirac-Kähler equation is shown. Moreover, in the massive case we prove that a discrete Dirac-Kähler operator flips the chirality.
\end{abstract}

Keywords: Dirac-Kähler equation, chirality, Hodge star operator, difference equations, discrete models.

Math. Subj. Clas.: 39A12, 39A70, 35Q41

\section{Introduction}

This work is a direct continuation of the paper [17 in which we constructed a new discrete analogue of the Dirac-Kähler equation. In [17] we proposed a geometric discretisation scheme based on the formalism of differential forms and showed that many of the algebraic relations amongst the Hodge star operator *, the exterior product $\wedge$, the differential $d$, and the adjoint $\delta$ of $d$ that hold in the smooth setting also hold in the discrete case. This approach was originated by Dezin [6. There are alternative geometric discretisation schemes which base on the use of the differential form language [1, 3, 4, 7, 8, 14, 16, 19. Difficulties of the discretisation of the Hodge operators have been described by several authors [3, 14, 16, 21, 22, 23.

In this paper we are going to discuss a decomposition of the discrete DiracKähler equation into its self-dual and anti-self-dual parts. The reason that 
we did not consider this problem in [17] is that the Hodge star operator * and its discrete analogue are slightly differ. In the continual case the operator $(*)^{2}$ is either an involution or antiinvolution while in the discrete model $(*)^{2}$ is equivalent to a shift with corresponding sign. This is one of the main distinctive features of the formalism [17] as compared to the continual case. Now we define a discrete star operator using a combinatorial double complex construction. In this way we obtain the operator which is more like its continual analogue since $(*)^{2}= \pm I$, where $I$ is the identity operator. At the same time in the double complex we will use discrete analogues of differential forms, $d$, and $\wedge$ defined in [17. The discrete star operator proposed here still preserves the Lorentz metric structure in our discrete model. It make possible to define a discrete analogue of $\delta$ by using an inner product of discrete forms (cochains) which imitate the continual case.

From the physics point of view self-dual and anti-self-dual fields of Dirac theory correlate with chiral fermions. It is well known that the chirality is an important feature of the Dirac theory. However, in lattice formulations of fermions the chirality problem is one of the most notorious. This problem deals with breaking chiral symmetry on the lattice [5, 9, 12, 13, 20. See also more resent work [10]. For the Dirac-Kähler equation on the lattice this difficulty was discussed first by Rabin [14. In 22 a discretisation scheme in the Dirac-Kähler setting was proposed in which the chirality is captured on the lattice. Chiral fermions can be described by using the fifth gamma matrix: $\gamma^{5}=\mathrm{i} \gamma^{0} \gamma^{1} \gamma^{2} \gamma^{3}$. The projection operators $P_{L}=\frac{I-\gamma^{5}}{2}$ and $P_{R}=\frac{I+\gamma^{5}}{2}$ decompose any Dirac field into its left-handed and right-handed parts. Rabin [14 pointed out that in the language of differential forms the chiral symmetry is a rotation of mixing forms with their duals and the Hodge star operator plays a central role here. However, the operator $*$ is somewhat different from $\gamma^{5}$. Therefore, we introduce a modified star operator which plays a role of $\gamma^{5}$ in our discrete model. Self-dual and anti-self-dual discrete inhomogeneous forms with respect to this new star operator are considered. From the viewpoint presented here this allows us to deal with chirality. We show that, just as in the continuum case, a discrete massless Dirac-Kähler equation admits the chiral invariance. We prove also that in the massive case a discrete analog of the Dirac-Kähler operator flips the chirality.

We first briefly review some definitions and basic notations on the DiracKähler equation [11, 14]. Let $M=\mathbb{R}^{1,3}$ be Minkowski space with metric signature $(+,-,-,-)$. Denote by $\Lambda^{r}(M)$ the vector space of smooth differential $r$-form, $r=0,1,2,3,4$. We consider $\Lambda^{r}(M)$ over $\mathbb{C}$. Let $\omega$ and $\varphi$ be complexvalued $r$-forms on $M$. The inner product is defined by

$$
(\omega, \varphi)=\int_{M} \omega \wedge * \bar{\varphi},
$$

where $\wedge$ is the exterior product and $*$ is the Hodge star operator $*: \Lambda^{r}(M) \rightarrow$ $\Lambda^{4-r}(M)$ (with respect to the Lorentz metric). Let $d: \Lambda^{r}(M) \rightarrow \Lambda^{r+1}(M)$ be the exterior differential and let $\delta: \Lambda^{r}(M) \rightarrow \Lambda^{r-1}(M)$ be the formal adjoint of 
$d$ with respect to (1.1) (codifferential). We have $\delta=* d *$. Then the Laplacian (Laplace-Beltrami operator) acting on $r$-forms is defined by

$$
\Delta \equiv-d \delta-\delta d: \Lambda^{r}(M) \rightarrow \Lambda^{r}(M) .
$$

It is clear that

$$
-d \delta-\delta d=(d-\delta)^{2}=-(d+\delta)^{2} .
$$

Hence, a square root of the Laplacian can be written in two ways, namely either $d-\delta$ or $\mathrm{i}(d+\delta)$, where $\mathrm{i}$ is the usual complex unit $\left(\mathrm{i}^{2}=-1\right)$. In this paper we formulate the complex Dirac-Kähler equation by using the operator $\mathrm{i}(d+\delta)$. Denote by $\Lambda(M)$ the set of all differential forms on $M$. We have $\Lambda(M)=\Lambda^{0}(M) \oplus \Lambda^{1}(M) \oplus \Lambda^{2}(M) \oplus \Lambda^{3}(M) \oplus \Lambda^{4}(M)$. Let $\Omega \in \Lambda(M)$ be an inhomogeneous differential form. This form can be expanded as

$$
\Omega=\sum_{r=0}^{4} \stackrel{r}{\omega}
$$

where $\stackrel{r}{\omega} \in \Lambda^{r}(M)$. The Dirac-Kähler equation is given by

$$
\mathrm{i}(d+\delta) \Omega=m \Omega,
$$

where $m$ is a mass parameter. It is easy to show that Eq. (1.3) is equivalent to the set of equations

$$
\begin{aligned}
& \mathrm{i} \delta \stackrel{1}{\omega}=m \stackrel{0}{\omega},
\end{aligned}
$$

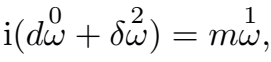

$$
\begin{aligned}
& \mathrm{i}(d \stackrel{1}{\omega}+\delta \stackrel{3}{\omega})=m \stackrel{2}{\omega}, \\
& \mathrm{i}(d \stackrel{2}{\omega}+\delta \stackrel{4}{\omega})=m \stackrel{3}{\omega}, \\
& \mathrm{i} d \stackrel{3}{\omega}=m \stackrel{4}{\omega} .
\end{aligned}
$$

\section{Double complex construction}

In this section we introduce a double complex construction which bases on the combinatorial model of Minkowski space described in [17]. For the convenience of the reader we briefly repeat the relevant material from [17] without proofs, thus making our presentation self-contained.

Let the tensor product $C(4)=C \otimes C \otimes C \otimes C$ of a 1-dimensional complex be a combinatorial model of Euclidean space $\mathbb{R}^{4}$. The 1-dimensional complex $C$ is defined in the following way. Let $C^{0}$ denotes the real linear space of 0 -dimensional chains generated by basis elements $x_{\kappa}$ (points), $\kappa \in \mathbb{Z}$. It is convenient to introduce the shift operator $\tau$ in the set of indices by

$$
\tau \kappa=\kappa+1 .
$$


We denote the open interval $\left(x_{\kappa}, x_{\tau \kappa}\right)$ by $e_{\kappa}$. One can regard the set $\left\{e_{\kappa}\right\}$ as a set of basis elements of the real linear space $C^{1}$. Suppose that $C^{1}$ is the space of 1-dimensional chains. Then the 1-dimensional complex (combinatorial real line) is the direct sum of the introduced spaces $C=C^{0} \oplus C^{1}$. The boundary operator $\partial$ in $C$ is given by

$$
\partial x_{\kappa}=0, \quad \partial e_{\kappa}=x_{\tau \kappa}-x_{\kappa} .
$$

The definition is extended to arbitrary chains by linearity.

Multiplying the basis elements $x_{\kappa}, e_{\kappa}$ in various way we obtain basis elements of $C(4)$. Let $s_{k}$ be an arbitrary basis element of $C(4)$. Then we have $s_{k}=s_{k_{0}} \otimes s_{k_{1}} \otimes s_{k_{2}} \otimes s_{k_{3}}$, where $s_{k_{i}}$ is either $x_{k_{i}}$ or $e_{k_{i}}$ and $k_{i} \in \mathbb{Z}$. Here $k=\left(k_{0}, k_{1}, k_{2}, k_{3}\right)$ is a multi-index. Let

$$
x_{k}=x_{k_{0}} \otimes x_{k_{1}} \otimes x_{k_{2}} \otimes x_{k_{3}} \quad \text { and } \quad e_{k}=e_{k_{0}} \otimes e_{k_{1}} \otimes e_{k_{2}} \otimes e_{k_{3}}
$$

be the 0 - and 4-dimensional basis elements of $C(4)$. The 1-dimensional basis elements of $C(4)$ can be written as

$$
\begin{array}{ll}
e_{k}^{0}=e_{k_{0}} \otimes x_{k_{1}} \otimes x_{k_{2}} \otimes x_{k_{3}}, & e_{k}^{1}=x_{k_{0}} \otimes e_{k_{1}} \otimes x_{k_{2}} \otimes x_{k_{3}}, \\
e_{k}^{2}=x_{k_{0}} \otimes x_{k_{1}} \otimes e_{k_{2}} \otimes x_{k_{3}}, & e_{k}^{3}=x_{k_{0}} \otimes x_{k_{1}} \otimes x_{k_{2}} \otimes e_{k_{3}},
\end{array}
$$

where the superscript $i$ indicates a place of $e_{k_{i}}$ in $e_{k}^{i}$ and $i=0,1,2,3$. In the same way we will write the 2-dimensional basis elements of $C(4)$ as

$$
\begin{array}{ll}
e_{k}^{01}=e_{k_{0}} \otimes e_{k_{1}} \otimes x_{k_{2}} \otimes x_{k_{3}}, & e_{k}^{12}=x_{k_{0}} \otimes e_{k_{1}} \otimes e_{k_{2}} \otimes x_{k_{3}}, \\
e_{k}^{02}=e_{k_{0}} \otimes x_{k_{1}} \otimes e_{k_{2}} \otimes x_{k_{3}}, & e_{k}^{13}=x_{k_{0}} \otimes e_{k_{1}} \otimes x_{k_{2}} \otimes e_{k_{3}}, \\
e_{k}^{03}=e_{k_{0}} \otimes x_{k_{1}} \otimes x_{k_{2}} \otimes e_{k_{3}}, & e_{k}^{23}=x_{k_{0}} \otimes x_{k_{1}} \otimes e_{k_{2}} \otimes e_{k_{3}} .
\end{array}
$$

Finally, denote by $e_{k}^{012}, e_{k}^{013}, e_{k}^{023}, e_{k}^{123}$ the 3 -dimensional basis elements of $C(4)$.

Let $C(4)=C(p) \otimes C(q)$, where $p+q=4$. If $a \in C(p)$ and $b \in C(q)$ are arbitrary chains, belonging to the complexes being multiplied, then we extend the definition of $\partial$ to chains of $C(4)$ by the rule

$$
\partial(a \otimes b)=\partial a \otimes b+(-1)^{r} a \otimes \partial b,
$$

where $r$ is the dimension of the chain $a$. It is easy to check that $\partial \partial a=0$.

Let us introduce the construction of a double complex. This construction generalizes that of [18. Together with the complex $C(4)$ we consider its double, namely the complex $\tilde{C}(4)$ of exactly the same structure. Define the one-to-one correspondence

$$
*^{c}: C(4) \rightarrow \tilde{C}(4), \quad *^{c}: \tilde{C}(4) \rightarrow C(4)
$$

in the following way. Let $s_{k}^{(r)}$ be an arbitrary $r$-dimensional basis element of $C(4)$, i.e., the product $s_{k}^{(r)}=s_{k_{0}} \otimes s_{k_{1}} \otimes s_{k_{2}} \otimes s_{k_{3}}$ contains exactly $r$ of 1dimensional elements $e_{k_{i}}$ and $4-r$ of 0 -dimensional elements $x_{k_{i}}$, and the 
superscript $(r)$ indicates a position of $e_{k_{i}}$ in $s_{k}^{(r)}$ (see, for example, (2.2) and (2.3)). Then

$$
*^{c} s_{k}^{(r)}=\varepsilon(r) \tilde{s}_{k}^{(4-r)}, \quad *^{c} \tilde{s}_{k}^{(r)}=\varepsilon(r) s_{k}^{(4-r)},
$$

where

$$
\tilde{s}_{k}^{(4-r)}=*^{c} s_{k_{0}} \otimes *^{c} s_{k_{1}} \otimes *^{c} s_{k_{2}} \otimes *^{c} s_{k_{3}}
$$

and $*^{c} s_{k_{i}}=\tilde{e}_{k_{i}}$ if $s_{k_{i}}=x_{k_{i}}$ and $*^{c} s_{k_{i}}=\tilde{x}_{k_{i}}$ if $s_{k_{i}}=e_{k_{i}}$. Here $\varepsilon(r)$ is the Levi-Civita symbol, i.e.,

$$
\varepsilon(r)=\left\{\begin{array}{lll}
+1 & \text { if }((r),(4-r)) & \text { is an even permutation of }(0,1,2,3) \\
-1 & \text { if }((r),(4-r)) & \text { is an odd permutation of }(0,1,2,3)
\end{array}\right.
$$

For example, for the 1- and 2-dimensional basis elements we have

$$
*^{c} e_{k}^{0}=\tilde{e}_{k}^{123}, \quad *^{c} e_{k}^{1}=-\tilde{e}_{k}^{023}, \quad *^{c} e_{k}^{2}=\tilde{e}_{k}^{013}, \quad *^{c} e_{k}^{3}=-\tilde{e}_{k}^{012}
$$

and

$$
\begin{array}{lll}
*^{c} e_{k}^{01}=\tilde{e}_{k}^{23}, & *^{c} e_{k}^{02}=-\tilde{e}_{k}^{13}, & *^{c} e_{k}^{03}=\tilde{e}_{k}^{12}, \\
*^{c} e_{k}^{12}=\tilde{e}_{k}^{03}, & *^{c} e_{k}^{13}=-\tilde{e}_{k}^{02}, & *^{c} e_{k}^{23}=\tilde{e}_{k}^{01} .
\end{array}
$$

We will also use the symbol $\varepsilon^{\prime}(r)$ to denote the Levi-Civita symbol given by

$$
\varepsilon^{\prime}(r)=\left\{\begin{array}{lll}
+1 & \text { if } \quad((4-r),(r)) & \text { is an even permutation of }(0,1,2,3) \\
-1 & \text { if } \quad((4-r),(r)) & \text { is an odd permutation of }(0,1,2,3) .
\end{array}\right.
$$

So we can write

$$
*^{c} s_{k}^{(4-r)}=\varepsilon^{\prime}(r) \tilde{s}_{k}^{(r)} \quad \text { and } \quad *^{c} \tilde{s}_{k}^{(4-r)}=\varepsilon^{\prime}(r) s_{k}^{(r)} .
$$

It is easy to check that

$$
\varepsilon(r) \varepsilon^{\prime}(r)=(-1)^{r} .
$$

Proposition 2.1. Let $a_{r} \in C(4)$ be an $r$-dimensional chain, i.e.,

$$
a_{r}=\sum_{k} \sum_{r} a_{(r)}^{k} s_{k}^{(r)}, \quad a_{(r)}^{k} \in \mathbb{R} .
$$

Then we have

$$
*^{c} *^{c} a_{r}=(-1)^{r} a_{r}
$$

Proof. The proof consists in applying the operation $*^{c}$ for basis elements and using (2.6).

Suppose that the combinatorial model of Minkowski space has the same structure as $C(4)$. We will use the index $k_{0}$ to denote the basis elements of $C$ which correspond to the time coordinate of $M$. Hence, the indicated basis elements will be written as $x_{k_{0}}, e_{k_{0}}$. 
Let us now consider a dual complex to $C(4)$. We define its as the complex of cochains $K(4)$ with complex coefficients. The complex $K(4)$ has a similar structure, namely $K(4)=K \otimes K \otimes K \otimes K$, where $K$ is a dual complex to the 1-dimensional complex $C$. We will write the basis elements of $K$ as $x^{\kappa}$ and $e^{\kappa}$, $\kappa \in \mathbb{Z}$. Then an arbitrary $r$-dimensional basis element of $K(4)$ can be written as $s_{(r)}^{k}=s^{k_{0}} \otimes s^{k_{1}} \otimes s^{k_{2}} \otimes s^{k_{3}}$, where $s^{k_{i}}$ is either $x^{k_{i}}$ or $e^{k_{i}}$. Note that the superscript and the subscript change places, as compared $s_{(r)}^{k}$ with the corresponding basis element $s_{k}^{(r)}$ of $C(4)$. We will call cochains forms, emphasizing their relationship with the corresponding continual objects, differential forms.

Denote by $K^{r}(4)$ the set of forms of degree $r$. We can represent $K(4)$ as

$$
K(4)=K^{0}(4) \oplus K^{1}(4) \oplus K^{2}(4) \oplus K^{3}(4) \oplus K^{4}(4) .
$$

Let $\stackrel{r}{\omega} \in K^{r}(4)$. Then we have

$$
\begin{gathered}
\stackrel{0}{\omega}=\sum_{k} \stackrel{0}{\omega}_{k} x^{k}, \quad \stackrel{4}{\omega}=\sum_{k} \stackrel{4}{\omega}_{k} e^{k}, \\
\stackrel{1}{\omega}=\sum_{k} \sum_{i=0}^{3} \omega_{k}^{i} e_{i}^{k}, \quad \stackrel{2}{\omega}=\sum_{k} \sum_{i<j} \omega_{k}^{i j} e_{i j}^{k}, \quad \stackrel{3}{\omega}=\sum_{k} \sum_{i<j<l} \omega_{k}^{i j l} e_{i j l}^{k},
\end{gathered}
$$

where the components $\stackrel{0}{\omega}_{k}, \stackrel{4}{\omega}_{k}, \omega_{k}^{i}, \omega_{k}^{i j}$ and $\omega_{k}^{i j l}$ are complex numbers.

As in [6] and [17, we define the pairing (chain-cochain) operation for any basis elements $\varepsilon_{k} \in C(4), s^{k} \in K(4)$ by the rule

$$
\left\langle\varepsilon_{k}, s^{k}\right\rangle= \begin{cases}0, & \varepsilon_{k} \neq s_{k} \\ 1, & \varepsilon_{k}=s_{k}\end{cases}
$$

The operation (2.11) is linearly extended to arbitrary chains and cochains.

The coboundary operator $d^{c}: K^{r}(4) \rightarrow K^{r+1}(4)$ is defined by

$$
\langle\partial a, \stackrel{r}{\omega}\rangle=\left\langle a, d^{c} \stackrel{r}{\omega}\right\rangle
$$

where $a \in C(4)$ is an $r+1$ dimensional chain. The operator $d^{c}$ is an analog of the exterior differential. From the above it follows that

$$
d^{c} \stackrel{4}{\omega}=0 \quad \text { and } \quad d^{c} d^{c} \stackrel{r}{\omega}=0 \text { for any } r .
$$

Let the difference operator $\Delta_{i}$ is given by

$$
\Delta_{i} \omega_{k}=\omega_{\tau_{i} k}-\omega_{k}
$$

for any components $\omega_{k} \in \mathbb{C}$ of $\stackrel{r}{\omega} \in K^{r}(4)$. For simplicity of notation we write here $\omega_{k}$ instead of $\omega_{k}^{(r)}$ and $\tau_{i}$ is the shift operator which acts as

$$
\tau_{i} k=\left(k_{0}, \ldots \tau k_{i}, \ldots k_{3}\right), \quad i=0,1,2,3,
$$


where $\tau$ is defined by (2.1). Using (2.4) and (2.12) we can calculate

$$
\begin{gathered}
d^{c} \stackrel{0}{\omega}=\sum_{k} \sum_{i=0}^{3}\left(\Delta_{i} \stackrel{0}{\omega}_{k}\right) e_{i}^{k}, \\
d^{c} \stackrel{1}{\omega}=\sum_{k} \sum_{i<j}\left(\Delta_{i} \omega_{k}^{j}-\Delta_{j} \omega_{k}^{i}\right) e_{i j}^{k}, \\
d^{c} \stackrel{2}{\omega}=\sum_{k}\left[\left(\Delta_{0} \omega_{k}^{12}-\Delta_{1} \omega_{k}^{02}+\Delta_{2} \omega_{k}^{01}\right) e_{012}^{k}\right. \\
+\left(\Delta_{0} \omega_{k}^{13}-\Delta_{1} \omega_{k}^{03}+\Delta_{3} \omega_{k}^{01}\right) e_{013}^{k} \\
+\left(\Delta_{0} \omega_{k}^{23}-\Delta_{2} \omega_{k}^{03}+\Delta_{3} \omega_{k}^{02}\right) e_{023}^{k} \\
\left.+\left(\Delta_{1} \omega_{k}^{23}-\Delta_{2} \omega_{k}^{13}+\Delta_{3} \omega_{k}^{12}\right) e_{123}^{k}\right], \\
d^{c} \stackrel{3}{\omega}=\sum_{k}\left(\Delta_{0} \omega_{k}^{123}-\Delta_{1} \omega_{k}^{023}+\Delta_{2} \omega_{k}^{013}-\Delta_{3} \omega_{k}^{012}\right) e^{k} .
\end{gathered}
$$

We now consider a multiplication of discrete forms which is an analog of the exterior multiplication for differential forms. Denote by $\cup$ this multiplication. For the basis elements of the one-dimensional complex $K(1)=K$ the $\cup$-multiplication is defined as follows

$$
x^{\kappa} \cup x^{\kappa}=x^{\kappa}, \quad e^{\kappa} \cup x^{\tau \kappa}=e^{\kappa}, \quad x^{\kappa} \cup e^{\kappa}=e^{\kappa}, \quad \kappa \in \mathbb{Z},
$$

supposing the product to be zero in all other case. To arbitrary basis element of $K(p)$ this definition is extended by induction on $p$, where $p=2,3,4$. See [6, 17] for details. The $\cup$-multiplication can be spread linearly to forms. This definition leads to the following discrete version of the Leibniz rule for differential forms.

Proposition 2.2. Let $\varphi$ and $\psi$ be arbitrary forms of K(4). Then

$$
d^{c}(\varphi \cup \psi)=d^{c} \varphi \cup \psi+(-1)^{r} \varphi \cup d^{c} \psi,
$$

where $r$ is the degree of a form $\varphi$.

Let $\tilde{K}(4)$ be a complex of the cochains over the double complex $\tilde{C}(4)$, with the coboundary operator $d^{c}$ defined in it by (2.12). Hence, $\tilde{K}(4)$ has the same structure as $K(4)$. The definitions of $d^{c}$ and $\cup$ do not depend on a metric. At the same time, to define a discrete analog of the Hodge star operator * we must take into account the Lorentz metric structure on $K(4)$. This means that Definition (2.5) is not suitable for a discrete version of the Hodge star operator. Define the operation $*: K^{r}(4) \rightarrow \tilde{K}^{4-r}(4)$ for an arbitrary basis element $s_{(r)}^{k}=s^{k_{0}} \otimes s^{k_{1}} \otimes s^{k_{2}} \otimes s^{k_{3}}$ by the rule

$$
* s_{(r)}^{k}=Q\left(k_{0}\right) \varepsilon(r) \tilde{s}_{(4-r)}^{k},
$$


where

$$
Q\left(k_{0}\right)=\left\{\begin{array}{lll}
+1 & \text { if } & s^{k_{0}}=x^{k_{0}} \\
-1 & \text { if } & s^{k_{0}}=e^{k_{0}}
\end{array}\right.
$$

This definition makes sense because the formula (2.17) preserves the Lorentz signature of metric in our discrete model. From (2.17) we obtain

$$
\begin{gathered}
* x^{k}=*\left(x^{k_{0}} \otimes x^{k_{1}} \otimes x^{k_{2}} \otimes x^{k_{3}}\right)=\tilde{e}^{k}, \\
* e^{k}=*\left(e^{k_{0}} \otimes e^{k_{1}} \otimes e^{k_{2}} \otimes e^{k_{3}}\right)=-\tilde{x}^{k}, \\
* e_{0}^{k}=-\tilde{e}_{123}^{k}, \quad * e_{1}^{k}=-\tilde{e}_{023}^{k}, \quad * e_{2}^{k}=\tilde{e}_{013}^{k}, \quad * e_{3}^{k}=-\tilde{e}_{012}^{k}, \\
* e_{01}^{k}=-\tilde{e}_{23}^{k}, \quad * e_{02}^{k}=\tilde{e}_{13}^{k}, \quad * e_{03}^{k}=-\tilde{e}_{12}^{k}, \\
* e_{12}^{k}=\tilde{e}_{03}^{k}, \quad * e_{13}^{k}=-\tilde{e}_{02}^{k}, \quad * e_{23}^{k}=\tilde{e}_{01}^{k}, \\
* e_{012}^{k}=-\tilde{e}_{3}^{k}, \quad * e_{013}^{k}=\tilde{e}_{2}^{k}, \quad * e_{023}^{k}=-\tilde{e}_{1}^{k}, \quad * e_{123}^{k}=-\tilde{e}_{0}^{k} .
\end{gathered}
$$

For any $r$-form we extend (2.17) by linearity.

It is easy to check that

$$
* * s_{(r)}^{k}=(-1)^{r(4-r)+1} s_{(r)}^{k}=(-1)^{r+1} s_{(r)}^{k} .
$$

Consequently, for each $r$-form $\stackrel{r}{\omega} \in K^{r}(4)$ we have

$$
* * \stackrel{r}{\omega}=(-1)^{r(4-r)+1} \stackrel{r}{\omega}=(-1)^{r+1} \stackrel{r}{\omega} .
$$

It means that the discrete $*$ operation imitates correctly the continual case.

Proposition 2.3. Let $a_{r} \in C(4)$ be an $r$-dimensional chain (2.7). Then we have

$$
\left\langle\tilde{a}_{r}, * \omega\right\rangle=(-1)^{r} Q\left(k_{0}\right)\left\langle *^{c} \tilde{a}_{r}, \omega\right\rangle
$$

where $\omega \in K^{4-r}(4)$.

Proof. The operations $*^{c}$ and $*$ are linear. It suffices to prove (2.24) for basis elements. Let $s_{(r)}^{k} \in K^{r}(4)$ be an arbitrary $r$-dimensional basis element of $K(4)$ which corresponds to $s_{k}^{(r)} \in C(4)$. By the definition of $*^{c}$ and by (2.11) we have

$$
1=\left\langle s_{k}^{(4-r)}, s_{(4-r)}^{k}\right\rangle=\varepsilon(r)\left\langle *^{c} \tilde{s}_{k}^{(r)}, s_{(4-r)}^{k}\right\rangle .
$$

On the other hand:

$$
1=\left\langle\tilde{s}_{k}^{(r)}, \tilde{s}_{(r)}^{k}\right\rangle=Q\left(k_{0}\right) \varepsilon^{\prime}(r)\left\langle\tilde{s}_{k}^{(r)}, * s_{(4-r)}^{k}\right\rangle
$$

which yields

$$
Q\left(k_{0}\right) \varepsilon^{\prime}(r)\left\langle\tilde{s}_{k}^{(r)}, * s_{(4-r)}^{k}\right\rangle=\varepsilon(r)\left\langle *^{c} \tilde{s}_{k}^{(r)}, s_{(4-r)}^{k}\right\rangle .
$$

Multiplying by $Q\left(k_{0}\right) \varepsilon^{\prime}(r)$ both sides of the above we find

$$
\left\langle\tilde{s}_{k}^{(r)}, * s_{(4-r)}^{k}\right\rangle=Q\left(k_{0}\right) \varepsilon^{\prime}(r) \varepsilon(r)\left\langle *^{c} \tilde{s}_{k}^{(r)}, s_{(4-r)}^{k}\right\rangle,
$$

since $Q^{2}\left(k_{0}\right)=\left(\varepsilon^{\prime}(r)\right)^{2}=1$. Thus, using (2.6) we obtain (2.24). 
Let $V \subset C(4)$ be a finite set of 4-dimensional basis elements of the complex $C(4)$. Define $V$ as follows

$$
V=\sum_{k} e_{k}, \quad k=\left(k_{0}, k_{1}, k_{2}, k_{3}\right), \quad k_{i}=1,2, \ldots, N_{i},
$$

where $N_{i} \in \mathbb{N}$ is a fixed number and $i=0,1,2,3$. We agree that in what follows the subscripts $k_{i}$ always run the set of values indicated in (2.25).

We set

$$
V_{r}=\sum_{k} \sum_{(r)} s_{k}^{(r)} \otimes *^{c} s_{k}^{(r)}
$$

For example,

$$
V_{1}=\sum_{k} \sum_{i=0}^{3} e_{k}^{i} \otimes *^{c} e_{k}^{i}=\sum_{k}\left(e_{k}^{0} \otimes \tilde{e}_{k}^{123}-e_{k}^{1} \otimes \tilde{e}_{k}^{023}+e_{k}^{2} \otimes \tilde{e}_{k}^{013}-e_{k}^{3} \otimes \tilde{e}_{k}^{012}\right)
$$

and

$V_{2}=\sum_{k} \sum_{i<j}^{3} e_{k}^{i j} \otimes *^{c} e_{k}^{i j}=\sum_{k}\left(e_{k}^{01} \otimes \tilde{e}_{k}^{23}-e_{k}^{02} \otimes \tilde{e}_{k}^{13}+e_{k}^{03} \otimes \tilde{e}_{k}^{12}+e_{k}^{12} \otimes \tilde{e}_{k}^{03}-e_{k}^{13} \otimes \tilde{e}_{k}^{02}+e_{k}^{23} \otimes \tilde{e}_{k}^{01}\right)$,

where $e_{k}^{i}$ and $e_{k}^{i j}$ are given by (2.2) and (2.3).

Let

$$
\mathbb{V}=\sum_{r=0}^{4} V_{r}
$$

For any $r$-forms $\varphi, \omega \in K^{r}(4)$ we define the inner product $(,)_{V}$ by

$$
\begin{aligned}
(\varphi, \omega)_{V} & =\langle\mathbb{V}, \varphi \otimes * \bar{\omega}\rangle=\left\langle V_{r}, \varphi \otimes * \bar{\omega}\right\rangle \\
& =\sum_{k} \sum_{(r)}\left\langle s_{k}^{(r)}, \varphi\right\rangle\left\langle *^{c} s_{k}^{(r)}, * \bar{\omega}\right\rangle,
\end{aligned}
$$

where $\bar{\omega}$ denotes the complex conjugate of the form $\omega$, i. e., $\bar{\omega}=\sum_{k} \sum_{(r)} \bar{\omega}_{k}^{(r)} s_{(r)}^{k}$. For the forms of different degrees the product (2.26) is set equal to zero. For example, if $\stackrel{1}{\varphi}, \stackrel{1}{\omega} \in K^{1}(4)$ then we obtain

$$
(\stackrel{1}{\varphi}, \stackrel{1}{\omega})_{V}=\sum_{k}\left[-\varphi_{k}^{0} \bar{\omega}_{k}^{0}+\varphi_{k}^{1} \bar{\omega}_{k}^{1}+\varphi_{k}^{2} \bar{\omega}_{k}^{2}+\varphi_{k}^{3} \bar{\omega}_{k}^{3}\right]
$$

It should be noted that in the definition of the inner product a role of $U$ multiplication is now played by the tensor multiplication (cf. 17]). In (2.26) the Lorentz matric structure is still captured. Using (2.11) and (2.18)-(2.22) 
we obtain

$$
\begin{aligned}
& (\stackrel{0}{\omega}, \stackrel{0}{\omega})_{V}=\sum_{k}\left|\stackrel{0}{\omega}_{k}\right|^{2}, \\
& (\stackrel{1}{\omega}, \stackrel{1}{\omega})_{V}=\sum_{k}\left(-\left|\omega_{k}^{0}\right|^{2}+\left|\omega_{k}^{1}\right|^{2}+\left|\omega_{k}^{2}\right|^{2}+\left|\omega_{k}^{3}\right|^{2}\right), \\
& (\stackrel{2}{\omega}, \stackrel{2}{\omega})_{V}=\sum_{k}\left(-\left|\omega_{k}^{01}\right|^{2}-\left|\omega_{k}^{02}\right|^{2}-\left|\omega_{k}^{03}\right|^{2}+\left|\omega_{k}^{12}\right|^{2}+\left|\omega_{k}^{13}\right|^{2}+\left|\omega_{k}^{23}\right|^{2}\right), \\
& (\stackrel{3}{\omega}, \stackrel{3}{\omega})_{V}=\sum_{k}\left(-\left|\omega_{k}^{012}\right|^{2}-\left|\omega_{k}^{013}\right|^{2}-\left|\omega_{k}^{023}\right|^{2}+\left|\omega_{k}^{123}\right|^{2}\right), \\
& (\stackrel{4}{\omega}, \stackrel{4}{\omega})_{V}=-\sum_{k}\left|\stackrel{4}{\omega}_{k}\right|^{2} .
\end{aligned}
$$

The inner product makes it possible to define the adjoint of $d^{c}$, denoted $\delta^{c}$.

Proposition 2.4. For any $(r-1)$-form $\varphi$ and $r$-form $\omega$ we have

$$
\left(d^{c} \varphi, \omega\right)_{V}=\langle\partial \mathbb{V}, \varphi \otimes * \bar{\omega}\rangle+\left(\varphi, \delta^{c} \omega\right)_{V},
$$

where

$$
\delta^{c}=(-1)^{r} *^{-1} d^{c} *
$$

and $* *^{-1}=I$.

Proof. The proof is a computation. From the definition (2.12) it follows that (2.4) induces the similar relation for the coboundary operator $d^{c}$ on forms:

$$
d^{c}(\varphi \otimes * \omega)=d^{c} \varphi \otimes * \omega+(-1)^{r-1} \varphi \otimes d^{c}(* \omega) .
$$

Using this we compute

$$
\begin{aligned}
\left(d^{c} \varphi, \omega\right)_{V} & =\left\langle\mathbb{V}, d^{c} \varphi \otimes * \bar{\omega}\right\rangle=\left\langle V_{r}, d^{c} \varphi \otimes * \bar{\omega}\right\rangle \\
& =\left\langle\mathbb{V}, d^{c}(\varphi \otimes * \bar{\omega})\right\rangle-(-1)^{r-1}\left\langle\mathbb{V}, \varphi \otimes d^{c}(* \bar{\omega})\right\rangle \\
& =\langle\partial \mathbb{V}, \varphi \otimes * \bar{\omega}\rangle+(-1)^{r}\left\langle\mathbb{V}, \varphi \otimes *\left(*^{-1} d^{c} * \bar{\omega}\right)\right\rangle \\
& =\langle\partial \mathbb{V}, \varphi \otimes * \bar{\omega}\rangle+\left\langle V_{r-1}, \varphi \otimes *\left(\delta^{c} \bar{\omega}\right)\right\rangle .
\end{aligned}
$$

It immediately follows (2.27).

Relation (2.27) is a discrete analog of the Green formula. From (2.23) we infer

$$
*^{-1}=(-1)^{r(4-r)+1} *=(-1)^{r+1} * .
$$

Putting this in (2.28) we obtain

$$
\delta^{c}=* d^{c} * .
$$

This makes it clear that the operator $\delta^{c}: K^{r+1}(4) \rightarrow K^{r}(4)$ is a discrete analog of the codifferential $\delta$. For the 0 -form (2.9) we have $\delta^{c} \stackrel{0}{\omega}=0$. Note that the 
difference expression for $\delta^{c}$ is slightly different than that given in [17]. Using (2.13) - (2.16) and (2.18)-(2.22) we can calculate

$$
\begin{gathered}
\delta^{c} \omega=\sum_{k}\left(\Delta_{0} \omega_{k}^{0}-\Delta_{1} \omega_{k}^{1}-\Delta_{2} \omega_{k}^{2}-\Delta_{3} \omega_{k}^{3}\right) x^{k}, \\
\delta^{c} \stackrel{\omega}{\omega}=\sum_{k}\left[\left(\Delta_{1} \omega_{k}^{01}+\Delta_{2} \omega_{k}^{02}+\Delta_{3} \omega_{k}^{03}\right) e_{0}^{k}\right. \\
+\left(\Delta_{0} \omega_{k}^{01}+\Delta_{2} \omega_{k}^{12}+\Delta_{3} \omega_{k}^{13}\right) e_{1}^{k} \\
+\left(\Delta_{0} \omega_{k}^{02}-\Delta_{1} \omega_{k}^{12}+\Delta_{3} \omega_{k}^{23}\right) e_{2}^{k} \\
\left.+\left(\Delta_{0} \omega_{k}^{03}-\Delta_{1} \omega_{k}^{13}-\Delta_{2} \omega_{k}^{23}\right) e_{3}^{k}\right], \\
\delta^{c} \stackrel{3}{\omega}=\sum_{k}\left[\left(-\Delta_{2} \omega_{k}^{012}-\Delta_{3} \omega_{k}^{013}\right) e_{01}^{k}+\left(\Delta_{1} \omega_{k}^{012}-\Delta_{3} \omega_{k}^{023}\right) e_{02}^{k}\right. \\
+\left(\Delta_{1} \omega_{k}^{013}+\Delta_{2} \omega_{k}^{023}\right) e_{03}^{k}+\left(\Delta_{0} \omega_{k}^{012}-\Delta_{3} \omega_{k}^{123}\right) e_{12}^{k} \\
\left.+\left(\Delta_{0} \omega_{k}^{013}+\Delta_{2} \omega_{k}^{123}\right) e_{13}^{k}+\left(\Delta_{0} \omega_{k}^{023}-\Delta_{1} \omega_{k}^{123}\right) e_{23}^{k}\right] \\
\delta^{c} \stackrel{4}{\omega}=\sum_{k}\left[\left(\Delta_{3}{ }^{4} \omega_{k}\right) e_{012}^{k}-\left(\Delta_{2} \omega_{k}^{4}\right) e_{013}^{k}\right. \\
\left.+\left(\Delta_{1} \stackrel{4}{\omega}_{k}\right) e_{023}^{k}+\left(\Delta_{0} \stackrel{4}{\omega}_{k}\right) e_{123}^{k}\right] .
\end{gathered}
$$

It is obvious that $\delta^{c} \delta^{c} \stackrel{r}{\omega}=0$ for any $r=1,2,3,4$. The linear map

$$
\Delta^{c}=-\left(d^{c} \delta^{c}+\delta^{c} d^{c}\right): K^{r}(4) \rightarrow K^{r}(4)
$$

is called a discrete analogue of the Laplacian (1.2). It is clear that

$$
-\left(d^{c} \delta^{c}+\delta^{c} d^{c}\right)=\left(d^{c}-\delta^{c}\right)^{2}=\mathrm{i}\left(d^{c}+\delta^{c}\right)^{2} .
$$

Finally, let us introduce the following operation

$$
\tilde{\iota}: K^{r}(4) \rightarrow \tilde{K}^{r}(4), \quad \tilde{\iota}: \tilde{K}^{r}(4) \rightarrow K^{r}(4)
$$

by setting

$$
\tilde{\iota} s_{(r)}^{k}=\tilde{s}_{(r)}^{k}, \quad \tilde{\iota} \tilde{s}_{(r)}^{k}=s_{(r)}^{k},
$$

where $s_{(r)}^{k}$ and $\tilde{s}_{(r)}^{k}$ are basis elements of $K^{r}(4)$ and $\tilde{K}^{r}(4)$ respectively. Consequently, for an $r$-form $\varphi \in K^{r}(4)$ we have $\tilde{\iota} \varphi=\tilde{\varphi}$. Recall that the components of $\tilde{\varphi} \in \tilde{K}^{r}(4)$ and $\varphi \in K^{r}(4)$ are the same.

Proposition 2.5. The following hold

$$
\tilde{\iota}^{2}=I, \quad \tilde{\iota} *=* \tilde{\iota}, \quad \tilde{\iota} d^{c}=d^{c} \tilde{\iota}, \quad \tilde{\iota} \delta^{c}=\delta^{c} \tilde{\iota} .
$$

Proof. The proof immediately follows from definitions of the corresponding operations. 


\section{Discrete Dirac-Kähler equation and chiral sym- metry}

Let us introduce a discrete inhomogeneous form as follows

$$
\Omega=\sum_{r=0}^{4} \stackrel{r}{\omega}
$$

where $\stackrel{r}{\omega}$ is given by (2.9) and (2.10). Due to (2.34) a discrete analog of the Dirac-Kähler equation (1.3) can be defined as

$$
\mathrm{i}\left(d^{c}+\delta^{c}\right) \Omega=m \Omega .
$$

We can write this equation more explicitly by separating its homogeneous components as

$$
\begin{aligned}
\mathrm{i} \delta^{c} \stackrel{1}{\omega} & =m \stackrel{0}{\omega}, \\
\mathrm{i}\left(d^{c} \stackrel{0}{\omega}+\delta^{c} \stackrel{1}{\omega}\right) & =m \stackrel{\omega}{\omega}, \\
\mathrm{i}\left(d^{c} \stackrel{1}{\omega}+\delta^{c} \stackrel{3}{\omega}\right) & =m \stackrel{2}{\omega}, \\
\mathrm{i}\left(d^{c} \stackrel{2}{\omega}+\delta^{c} \stackrel{4}{\omega}\right) & =m \stackrel{3}{\omega}, \\
\mathrm{i} d^{c} \stackrel{3}{\omega} & =m \stackrel{4}{\omega} .
\end{aligned}
$$


This set of equations can be expressed in terms of difference equations. Substituting (2.13)-(2.16) and (2.30)-(2.33) into (3.3) we obtain

$$
\begin{array}{r}
\mathrm{i}\left(\Delta_{0} \omega_{k}^{0}-\Delta_{1} \omega_{k}^{1}-\Delta_{2} \omega_{k}^{2}-\Delta_{3} \omega_{k}^{3}\right)=m \omega_{k}^{0}, \\
\mathrm{i}\left(\Delta_{0} \stackrel{0}{\omega}_{k}+\Delta_{1} \omega_{k}^{01}+\Delta_{2} \omega_{k}^{02}+\Delta_{3} \omega_{k}^{03}\right)=m \omega_{k}^{0}, \\
\mathrm{i}\left(\Delta_{1} \stackrel{\omega}{\omega}_{k}+\Delta_{0} \omega_{k}^{01}+\Delta_{2} \omega_{k}^{12}+\Delta_{3} \omega_{k}^{13}\right)=m \omega_{k}^{1}, \\
\mathrm{i}\left(\Delta_{2} \omega_{k}+\Delta_{0} \omega_{k}^{02}-\Delta_{1} \omega_{k}^{12}+\Delta_{3} \omega_{k}^{23}\right)=m \omega_{k}^{2}, \\
\mathrm{i}\left(\Delta_{3} \omega_{k}+\Delta_{0} \omega_{k}^{03}-\Delta_{1} \omega_{k}^{13}-\Delta_{2} \omega_{k}^{23}\right)=m \omega_{k}^{3}, \\
\mathrm{i}\left(\Delta_{0} \omega_{k}^{1}-\Delta_{1} \omega_{k}^{0}-\Delta_{2} \omega_{k}^{012}-\Delta_{3} \omega_{k}^{013}\right)=m \omega_{k}^{01}, \\
\mathrm{i}\left(\Delta_{0} \omega_{k}^{2}-\Delta_{2} \omega_{k}^{0}+\Delta_{1} \omega_{k}^{012}-\Delta_{3} \omega_{k}^{023}\right)=m \omega_{k}^{02}, \\
\mathrm{i}\left(\Delta_{0} \omega_{k}^{3}-\Delta_{3} \omega_{k}^{0}+\Delta_{1} \omega_{k}^{013}+\Delta_{2} \omega_{k}^{023}\right)=m \omega_{k}^{03}, \\
\mathrm{i}\left(\Delta_{1} \omega_{k}^{2}-\Delta_{2} \omega_{k}^{1}+\Delta_{0} \omega_{k}^{012}-\Delta_{3} \omega_{k}^{123}\right)=m \omega_{k}^{12}, \\
\mathrm{i}\left(\Delta_{1} \omega_{k}^{3}-\Delta_{3} \omega_{k}^{1}+\Delta_{0} \omega_{k}^{013}+\Delta_{2} \omega_{k}^{123}\right)=m \omega_{k}^{13}, \\
\mathrm{i}\left(\Delta_{2} \omega_{k}^{3}-\Delta_{3} \omega_{k}^{2}+\Delta_{0} \omega_{k}^{023}-\Delta_{1} \omega_{k}^{123}\right)=m \omega_{k}^{23}, \\
\mathrm{i}\left(\Delta_{0} \omega_{k}^{12}-\Delta_{1} \omega_{k}^{02}+\Delta_{2} \omega_{k}^{01}+\Delta_{3} \omega_{k}\right)=m \omega_{k}^{012}, \\
\mathrm{i}\left(\Delta_{0} \omega_{k}^{13}-\Delta_{1} \omega_{k}^{03}+\Delta_{3} \omega_{k}^{01}-\Delta_{2} \omega_{k}\right)=m \omega_{k}^{013}, \\
\mathrm{i}\left(\Delta_{0} \omega_{k}^{23}-\Delta_{2} \omega_{k}^{03}+\Delta_{3} \omega_{k}^{02}+\Delta_{1} \stackrel{4}{\omega}_{k}\right)=m \omega_{k}^{023}, \\
\mathrm{i}\left(\Delta_{1} \omega_{k}^{23}-\Delta_{2} \omega_{k}^{13}+\Delta_{3} \omega_{k}^{12}+\Delta_{0} \stackrel{4}{\omega}_{k}\right)=m \omega_{k}^{123}, \\
\mathrm{i}\left(\Delta_{0} \omega_{k}^{123}-\Delta_{1} \omega_{k}^{023}+\Delta_{2} \omega_{k}^{013}-\Delta_{3} \omega_{k}^{012}\right)=m \omega_{k}^{4} .
\end{array}
$$

Let us introduce the modified star operator $\star$ on inhomogeneous forms (3.1) by the rule

$$
\star \Omega=\mathrm{i} * B \Omega,
$$

where $B$ is the main antiautomorphism (see [15] for more details) which acts on $\Omega$ according to

$$
B \Omega=\sum_{r=0}^{4}(-1)^{\frac{r(r-1)}{2}} \stackrel{r}{\omega} .
$$

Proposition 3.1. The operator $\star$ is an involution, i.e.,

$$
\star \star \Omega=\Omega .
$$

Proof. Acting twice with $* B$ on an $r$-form and using (2.23) we obtain

$$
* B * B \stackrel{r}{\omega}=(-1)^{\frac{r(r-1)}{2}} * B(\stackrel{r}{\omega})=(-1)^{\frac{r(r-1)}{2}}(-1)^{\frac{(4-r)(4-r-1)}{2}} * * \stackrel{r}{\omega}=-\stackrel{r}{\omega} .
$$


Proposition 3.2. Let $\Omega$ be an inhomogeneous form. Then we have

$$
\star\left(d^{c}+\delta^{c}\right) \Omega+\left(d^{c}+\delta^{c}\right) \star \Omega=0 .
$$

Proof. By (3.5) for a homogeneous component of $\Omega$ we have

$$
\star \stackrel{r}{\omega}=(-1)^{\frac{r(r-1)}{2}} \mathrm{i} * \stackrel{r}{\omega} .
$$

Let $r=1$. Then

$$
\begin{aligned}
\star\left(d^{c}+\delta^{c}\right) \stackrel{1}{\omega} & =-\mathrm{i} * d^{c} \stackrel{1}{\omega}+\mathrm{i} * \delta^{c} \stackrel{1}{\omega}=-\mathrm{i} * d^{c} * * \stackrel{1}{\omega}+\mathrm{i} * * d^{c} * \stackrel{1}{\omega} \\
& =-\delta^{c}(\mathrm{i} * \stackrel{1}{\omega})-d^{c}(\mathrm{i} * \stackrel{1}{\omega})=-\delta^{c} \star \stackrel{1}{\omega}-d^{c} \star \stackrel{1}{\omega}=-\left(\delta^{c}+d^{c}\right) \star \stackrel{1}{\omega} .
\end{aligned}
$$

In the same way we obtain

$$
\begin{aligned}
\star\left(d^{c}+\delta^{c}\right) \stackrel{2}{\omega} & =-\mathrm{i} * d^{c} \stackrel{2}{\omega}+\mathrm{i} * \delta^{c} \stackrel{2}{\omega}=\mathrm{i} * d^{c} * * \underset{2}{\omega}+\mathrm{i} * * d^{c} * \stackrel{2}{\omega} \\
& =\delta^{c}(\mathrm{i} * \stackrel{2}{\omega})+d^{c}(\mathrm{i} * \stackrel{2}{\omega})=-\delta^{c} \star \stackrel{2}{\omega}-d^{c} \star \stackrel{2}{\omega}=-\left(\delta^{c}+d^{c}\right) \star \stackrel{2}{\omega}
\end{aligned}
$$

and

$$
\star\left(d^{c}+\delta^{c}\right) \stackrel{3}{\omega}=-\left(\delta^{c}+d^{c}\right) \star \stackrel{3}{\omega} .
$$

Next

$$
\star\left(d^{c}+\delta^{c}\right) \stackrel{0}{\omega}=\star d^{c} \stackrel{0}{\omega}=-\mathrm{i} * d^{c} * * \stackrel{0}{\omega}=-\delta^{c}(\mathrm{i} * \stackrel{0}{\omega})=-\delta^{c} \star \stackrel{0}{\omega}
$$

and

$$
\star\left(d^{c}+\delta^{c}\right) \stackrel{4}{\omega}=\star \delta^{c} \stackrel{4}{\omega}=-\mathrm{i} * * d^{c} * \stackrel{4}{\omega}=-d^{c}(\mathrm{i} * \stackrel{4}{\omega})=-d^{c} \star \stackrel{4}{\omega} .
$$

Hence

$$
\star\left(d^{c}+\delta^{c}\right) \Omega=\sum_{r=0}^{4} \star\left(d^{c}+\delta^{c}\right) \stackrel{r}{\omega}=-\sum_{r=0}^{4}\left(d^{c}+\delta^{c}\right) \star \stackrel{r}{\omega}=-\left(d^{c}+\delta^{c}\right) \star \Omega .
$$

By virtue of (3.6) and (3.7) we can claim that the operator $\star$ plays the same role as the fifth gamma matrix $\gamma^{5}$ in continual Dirac theory. Therefore, we can consider the chirality of our discrete model with respect to this operator.

We say that an inhomogeneous form $\Omega$ is self-dual or anti-self-dual if

$$
\tilde{\iota} \star \Omega=\Omega \quad \text { or } \quad \tilde{\iota} \star \Omega=-\Omega,
$$

where $\tilde{\iota}$ is defined by (2.35). The first equation of (3.9) is equivalent to the following equations

$$
\tilde{\iota} \star \stackrel{r}{\omega}=\stackrel{4-r}{\omega},
$$

where $r=0,1,2,3,4$. Hence, from (3.8) for a self-dual form we obtain

$$
\tilde{\iota} * \stackrel{0}{\omega}=-i \stackrel{4}{\omega}, \quad \tilde{\iota} * \stackrel{1}{\omega}=-\mathrm{i}_{\omega}^{\omega}, \quad \tilde{\iota} * \stackrel{2}{\omega}=\mathrm{i}^{2}, \quad \tilde{\iota} * \stackrel{3}{\omega}=\mathrm{i} \stackrel{1}{\omega}, \quad \tilde{\iota} * \stackrel{4}{\omega}=-\mathrm{i} \stackrel{0}{\omega} .
$$

The same equations can be drawn for an anti-self-dual form. 
Proposition 3.3. If the self-dual or anti-self-dual form $\Omega$ is a solution of the discrete Dirac-Kähler equation, then $\Omega$ is trivial, i. e., $\Omega=0$.

Proof. Let $\Omega$ be a self-dual form. By (3.10) from the first and fifth equations of (3.3) we have

$$
\begin{aligned}
m \stackrel{0}{\omega}=\mathrm{i} \delta^{c} \stackrel{1}{\omega} & =\mathrm{i} * d^{c} * \stackrel{1}{\omega}=\mathrm{i} * d^{c}(-\tilde{\imath} \mathrm{i} \stackrel{3}{\omega})=\tilde{\iota} * d^{c} \stackrel{3}{\omega} \\
& =\tilde{\imath} *(-\mathrm{i} m \stackrel{0}{\omega})=-\mathrm{i} m(\tilde{\iota} * \stackrel{4}{\omega})=-m \stackrel{\omega}{\omega} .
\end{aligned}
$$

Here we used (2.36). Since $m>0$ we obtain $\stackrel{0}{\omega}=0$. It follows immediately that $\stackrel{4}{\omega}=0$. Using this, from the second and fourth equations of (3.3) we obtain again

$$
\begin{aligned}
& m \stackrel{1}{\omega}=\mathrm{i}\left(d^{c} \stackrel{0}{\omega}+\delta^{c} \stackrel{2}{\omega}\right)=\mathrm{i} * d^{c} * \stackrel{2}{\omega}=\mathrm{i} * d^{c}(\tilde{\iota} \mathrm{i} \stackrel{2}{\omega})=-\tilde{\iota} * d^{c} \stackrel{2}{\omega} \\
& =-\tilde{\iota} *\left(-\mathrm{i} m \stackrel{3}{\omega}-\delta^{c} \stackrel{4}{\omega}\right)=\mathrm{i} m(\tilde{\iota} * \stackrel{3}{\omega})=\mathrm{i}^{2} m \stackrel{1}{\omega}=-m \stackrel{1}{\omega} \text {. }
\end{aligned}
$$

Thus, $\stackrel{1}{\omega}=0$ and this yields $\stackrel{3}{\omega}=0$. Furthermore, according to the third equation of (3.3) we have $\stackrel{2}{\omega}=0$.

An inhomogeneous form $\Omega$ decomposes into its self-dual and anti-self-dual parts with respect to the action of $\star$ as follows

$$
\Omega=\Omega^{+}+\Omega^{-},
$$

where

$$
\Omega^{ \pm}=\frac{1}{2}(\Omega \pm \tilde{\iota} \star \Omega) .
$$

It is clear that $\Omega^{+}$is self-dual and $\Omega^{-}$is anti-self-dual. The self-dual and antiself-dual components of $\Omega$ correspond to the chiral right and chiral left parts of a Dirac fermion. Denote by $\stackrel{r}{\omega}^{ \pm}$a homogeneous component of $\Omega^{ \pm}$. Using (3.8) we can write these components more explicitly as

$$
\begin{array}{rlrl}
\stackrel{0}{\omega}^{ \pm}=\frac{1}{2}(\stackrel{0}{\omega} \pm \mathrm{i} \tilde{\iota} * \stackrel{4}{\omega}), & \stackrel{1}{\omega}^{ \pm} & =\frac{1}{2}\left(\frac{1}{\omega} \mp \mathrm{i} \tilde{\iota} * \stackrel{3}{\omega}\right), & \stackrel{2}{\omega}^{ \pm}=\frac{1}{2}\left(\frac{2}{\omega} \mp \mathrm{i} \tilde{\iota} * \stackrel{2}{\omega}\right), \\
\stackrel{3}{\omega}^{ \pm} & =\frac{1}{2}(\stackrel{3}{\omega} \pm \mathrm{i} \tilde{\iota} * \stackrel{1}{\omega}), & \stackrel{4}{\omega}^{ \pm}=\frac{1}{2}(\stackrel{4}{\omega} \pm \mathrm{i} \tilde{\iota} * \stackrel{0}{\omega}) .
\end{array}
$$

Proposition 3.4. If $\Omega$ is a solution of the massless discrete Dirac-Kähler equation

$$
\left(d^{c}+\delta^{c}\right) \Omega=0,
$$

then so do both $\Omega^{+}$and $\Omega^{-}$.

Proof. It suffices to prove the claim for one of Eqs. (3.3), say for the second one. Let

$$
\mathrm{i}\left(d^{c} \stackrel{0}{\omega}+\delta^{c} \stackrel{2}{\omega}\right)=0 .
$$


Then, by (3.11), for the corresponding homogeneous components of $\Omega^{+}$we obtain

$$
\begin{aligned}
\mathrm{i}\left(d^{c} \stackrel{0}{\omega}^{+}+\delta^{c} \stackrel{2}{\omega}^{+}\right) & =\frac{1}{2} \mathrm{i}\left(d^{c}(\stackrel{0}{\omega}+\mathrm{i} \tilde{\iota} * \stackrel{4}{\omega})+\delta^{c}(\stackrel{2}{\omega}-\mathrm{i} \tilde{\iota} * \stackrel{2}{\omega})\right) \\
& =\frac{1}{2} \mathrm{i}\left(d^{c} \stackrel{0}{\omega}+\delta^{c} \stackrel{2}{\omega}\right)+\frac{1}{2} \tilde{\iota}\left(-d^{c}(* \stackrel{4}{\omega})+\delta^{c}(* \stackrel{2}{\omega})\right) \\
& =\frac{1}{2} \tilde{\iota}\left(-* * d^{c} * \stackrel{4}{\omega}+* d^{c} * * \stackrel{2}{\omega}\right)=-\frac{1}{2} \tilde{\iota} *\left(\delta^{c} \stackrel{4}{\omega}+d^{c} \stackrel{2}{\omega}\right)=0 .
\end{aligned}
$$

Here we used (2.23), (2.29) and (2.36). The other cases are similar.

Proposition 3.4 gives rise to the chiral symmetry of our discrete model. This means that equation (3.12) is invariant under the transformation

$$
\Omega \longrightarrow \Omega \pm \tilde{\imath} \star \Omega \text {. }
$$

This transformation is equivalent to

$$
\stackrel{r}{\omega} \longrightarrow \stackrel{r}{\omega} \pm \tilde{\iota} \star \stackrel{4}{\omega}^{r}
$$

and the equation

$$
d^{c^{r-1}}{ }^{\frac{1}{\omega}}+\delta^{c+1} \omega=0
$$

is invariant under the transformation (3.13) for any $r=0,1,2,3,4$.

Proposition 3.5. If $\Omega$ is a solution of the discrete Dirac-Kähler equation (3.2) then we have

$$
\mathrm{i}\left(d^{c}+\delta^{c}\right) \Omega^{+}=m \Omega^{-}, \quad \mathrm{i}\left(d^{c}+\delta^{c}\right) \Omega^{-}=m \Omega^{+} .
$$

Proof. Substituting (3.11) into the first and second equations of (3.3) we obtain

$$
\begin{aligned}
& \mathrm{i} \delta^{c} \stackrel{1}{\omega}^{+}=\frac{1}{2} \mathrm{i}\left(\delta^{c} \stackrel{1}{\omega}-\mathrm{i} \tilde{\iota} \delta^{c} * \stackrel{3}{\omega}\right)=\frac{1}{2}\left(m \stackrel{0}{\omega}+\tilde{\iota} * d^{c} * * \stackrel{3}{\omega}\right)=\frac{1}{2}\left(m \stackrel{0}{\omega}+\tilde{\iota} * d^{c} \stackrel{3}{\omega}\right) \\
& =\frac{1}{2}(m \stackrel{0}{\omega}+\tilde{\iota} *(-\mathrm{i} m \stackrel{4}{\omega}))=\frac{1}{2} m(\stackrel{0}{\omega}-\mathrm{i} \tilde{\iota} * \stackrel{4}{\omega})=\frac{1}{2} m(\stackrel{0}{\omega}-\tilde{\iota} \star \stackrel{4}{\omega})=m \stackrel{0}{\omega}^{-}
\end{aligned}
$$

and

$$
\begin{aligned}
\mathrm{i}\left(d^{c} \stackrel{0}{\omega}^{+}+\delta^{c} \stackrel{2}{\omega}^{+}\right) & =\frac{1}{2} \mathrm{i} d^{c}(\stackrel{0}{\omega}+\mathrm{i} \tilde{\iota} * \stackrel{4}{\omega})+\frac{1}{2} \mathrm{i} \delta^{c}(\stackrel{2}{\omega}-i \tilde{\imath} * \stackrel{2}{\omega}) \\
& =\frac{1}{2}\left(m \stackrel{1}{\omega}-\tilde{\iota} d^{c} * \stackrel{4}{\omega}+\tilde{\iota} \delta^{c} * \stackrel{2}{\omega}\right)=\frac{1}{2}\left(m \stackrel{1}{\omega}-\tilde{\iota} * * d^{c} * \stackrel{4}{\omega}+\tilde{\imath} * d^{c} * * \stackrel{2}{\omega}\right) \\
& =\frac{1}{2}\left(m \stackrel{1}{\omega}-\tilde{\iota} * \delta^{c} \stackrel{4}{\omega}-\tilde{\iota} * d^{c} \stackrel{2}{\omega}\right)=\frac{1}{2}\left(m \stackrel{1}{\omega}-\tilde{\iota} *\left(\delta^{c} \stackrel{4}{\omega}+d^{c} \stackrel{2}{\omega}\right)\right) \\
& =\frac{1}{2}(m \stackrel{1}{\omega}-\tilde{\iota} *(-\mathrm{i} m \stackrel{3}{\omega}))=\frac{1}{2} m(\stackrel{1}{\omega}+\mathrm{i} \tilde{\iota} * \stackrel{3}{\omega})=\frac{1}{2} m(\stackrel{1}{\omega}-\tilde{\iota} \star \stackrel{3}{\omega})=m \stackrel{1}{\omega}^{-} .
\end{aligned}
$$

Here we used also (2.23), (2.29) and (3.8). Similar calculations give

$$
\mathrm{i}\left(d^{c} \stackrel{1}{\omega}^{+}+\delta^{c} 3^{+}\right)=m 2^{-}, \quad \mathrm{i}\left(d^{c} \underline{\omega}^{+}+\delta^{c} \dot{\omega}^{+}\right)=m 3^{-}, \quad \mathrm{i} d^{c^{3}} \stackrel{\omega}{\omega}^{+}=m \dot{4}^{-} .
$$

Consequently, the first equation of (3.14) is true. The remaining case is similar. 
Proposition 3.5 claims that in the massive case the operator i $\left(d^{c}+\delta^{c}\right)$ flips the chirality. It is known that in the continual Dirac theory the left-hand fermions turn into right-hand fermions after acting the Dirac operator and vice versa. Thus we have the same result in the discrete case.

\section{References}

[1] V. de Beaucé: Towards an algebraic approach to the discretisation of fermions., PoS LAT2005 (2005), 276.

[2] V. de Beaucé, S. Sen and J.C. Sexton: Chiral dirac fermions on the lattice using geometric discretisation, Nucl. Phys. B (Proc. Suppl.) 129-130 (2004), 468-470.

[3] P. Becher and H. Joos: The Dirac-Kähler equation and fermions on the lattice, Z. Phys. C 15 (1982), 343-365.

[4] S. Catterall: Dirac-Kähler fermions and exact lattice supersymmetry, PoS LAT2005 (2005), 006.

[5] S. Catterall, D. Kaplan and M. Ünsal: Exact lattice supersymmetry, Phys.Rept. 484 (2009), 71-130.

[6] A.A. Dezin: Multidimensional analysis and discrete models, CRC Press, Boca Raton, 1995.

[7] J. Dodziuk: Finite-difference approach to Hodge theory of harmonic forms, Amer. J. Math. 98 (1976), 79-104.

[8] I. Kanamori and N. Kawamoto: Dirac-Kähler fermion from Clifford product with noncommutative differential form on a lattice, Int. J. Mod. Phys. A 19(5), (2004), 695-736.

[9] D. Kaplan: A method for simulating chiral fermions on the lattice, Phys. Lett. B 288 (1992), 342-347.

[10] D. Kaplan: Chiral Symmetry and Lattice Fermions, arXiv: 0912.2560v2, (2012).

[11] E. Kähler: Der innere differentialkül, Rendiconti di Matematica 21(3-4) (1962), 425-523.

[12] M. Lüscher: Exact chiral symmetry on the lattice and the Ginsparg-Wilson relation, Phys.Lett. B 428(10) (1998), 342-345.

[13] R. Narayanan and H. Neuberger: A construction of lattice chiral gauge theories, Nucl. Phys. B 443 (1995), 305-385.

[14] J.M. Rabin: Homology theory of lattice fermion doubling, Nucl. Phys. B 201 (2) (1982), 315-332. 
[15] M. Reuter: Symplectic Dirac-Kähler fields, J. Math. Phys. 40 (1999), 55935640 .

[16] S. Sen, S. Sen, J.C. Sexton, and D. Adams: A geometric discretisation scheme applied to the Abelian Chern-Simons theory, Phys. Rev. E 61 (2000), 3174-3185.

[17] V. Sushch: A discrete model of the Dirac-Kähler equation. Rep. Math. Phys. 73(1) (2014), 109-125.

[18] V. Sushch: Self-dual and anti-self-dual solutions of discrete Yang-Mills equations on a double complex. Cubo 12(3) (2010), 99-120.

[19] V. Sushch: On some finite-difference analogs of invariant first-order hyperbolic systems, Diff. Equ. 35(3) (1999), 414-420; translation from Differ. Uravn. 35(3) (1999), 411-417.

[20] L. Susskind: Lattice fermions, Phys. Rev. D 16 (1977), 3031.

[21] F.L. Teixeira: Lattice Maxwell's Equations, Progress in Electromagnetics Research 148 (2014), 113-128.

[22] F.L. Teixeira and W.C. Chew: Lattice electromagnetic theory from a topological viewpoint, J. Math. Phys. 40(1) (1999), 168-187.

[23] S.O. Wilson: Differential forms, fluids, and finite models, Proc. of Amer. Math. Soc. 139 (2011), 2597-2604. 